\title{
Muséologies
}

Les cahiers d'études supérieures

\section{Étude des programmes éducatifs offerts au public dans les jardins historiques du Québec}

\section{Ève-Marie Proulx}

Volume 1, numéro 2, avril 2007

URI : https://id.erudit.org/iderudit/1033611ar

DOI : https://doi.org/10.7202/1033611ar

Aller au sommaire du numéro

Éditeur(s)

Association Québécoise de Promotion des Recherches Étudiantes en

Muséologie (AQPREM)

\section{ISSN}

1718-5181 (imprimé)

1929-7815 (numérique)

Découvrir la revue

Citer cet article

Proulx, È.-M. (2007). Étude des programmes éducatifs offerts au public dans les jardins historiques du Québec. Muséologies, 1(2), 106-121.

https://doi.org/10.7202/1033611ar
Résumé de l'article

L'auteur étudie l'influence de la nature particulière des jardins historiques sur les programmes éducatifs qui y sont proposés. Pour ce faire, elle définit des critères de classement des jardins et analyse les activités offertes selon leur nature et leur public cible, dans le but d'en identifier les points forts et les lacunes. 


\section{Étude des programmes éducatifs}

offerts au public dans les jardins

historiques du Québec

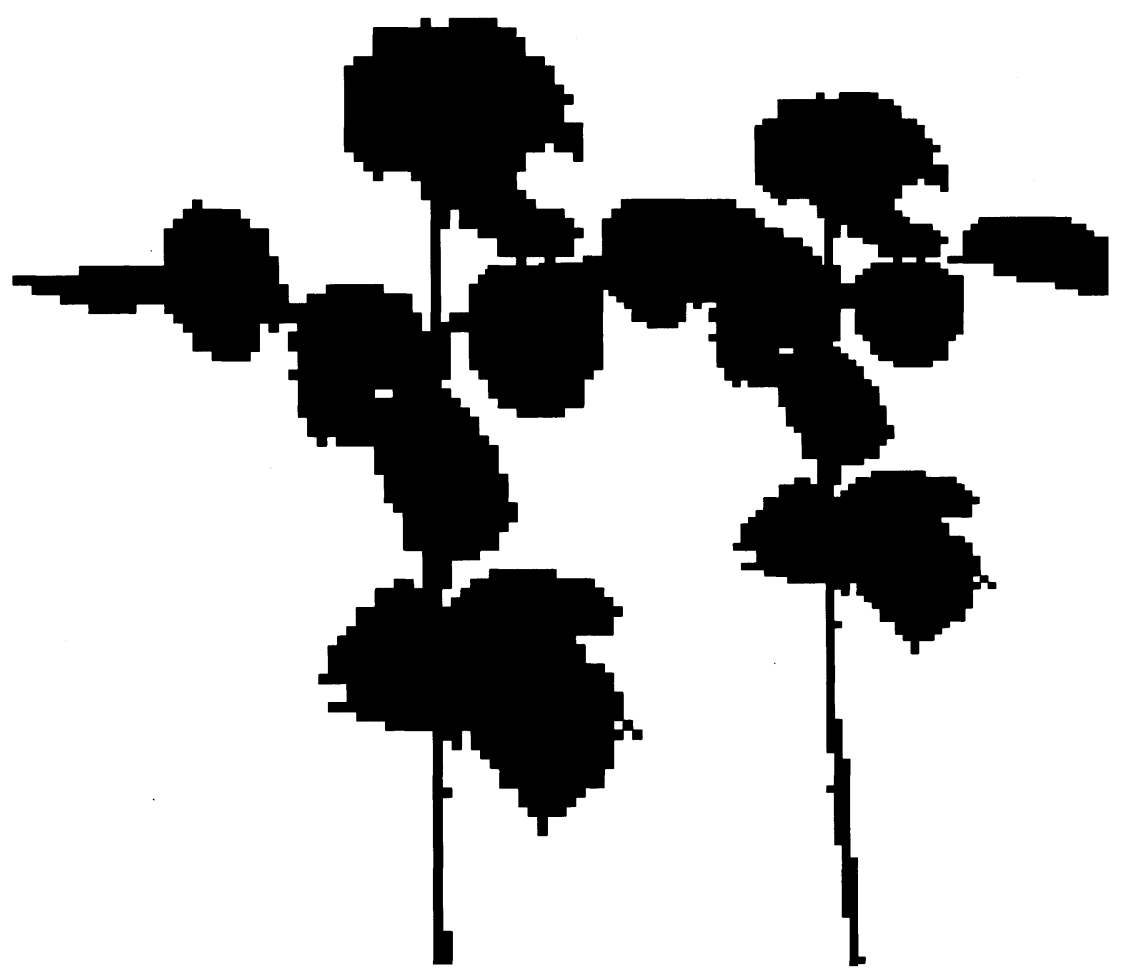


L'auteur étudie l'influence de la nature particulière des jardins historiques sur les programmes éducatifs qui y sont proposés. Pour ce faire, elle définit des critères de classement des jardins et analyse les activités offertes selon leur nature et leur public cible, dans le but d'en identifier les points forts et les lacunes.

ĖVE-MARIE PROULX A OBTENU UN BACCALAURÉAT EN GÉNIE CHIMIQUE EN 1999 ET UNE MAITTRISE EN MUSÉOLOGIE EN 2006. ELLE TRAVAILLE COMME INGÉNIEURE CONSULTANTE DANS LE DOMAINE DE LA MÉTALLURGIE ET DE L'ENVIRONNEMENT. À L'HIVER 2007, ELLE EST CHARGÉE D'UN COURS DE LA MAITTRISE EN MUSÉOLOGIE À L'UNIVERSITÉ DE MONTRÉAL PORTANT SUR LES COLLECTIONS DE SCIENCES NATURELLES ET LEUR MISE EN VALEUR. LARTICLE QUI SUIT PRÉSENTE LES RÉSULTATS DE SON TRAVAIL DIRIGÉ DE MAîTRISE QUI A ÉTÉ MIS EN NOMINATION POUR LE PRIX ROLAND-ARPIN EN 2006. 
MARTIN, P.-L. et P. MORISSET. Promenades dans les jardins anciens du Québec. Montréal : Boréal, 1996, p. 8.

[2]

Association des jardins du Québec. <http://www.associationdesjardinsduquebec.com> (consulté en décembre 2005).
Au Québec, le jardinage est devenu, au cours des dernières décennies, une activité de loisir très populaire. Cette activité, ancrée dans le présent, ne s'appuie cependant pas nécessairement sur une longue tradition horticole. Ainsi, selon Martin et Morisset, auteurs d'un livre sur les jardins anciens du Québec, «la plupart des gens croient qu'il n'existe pas de jardins historiques au Québec " ${ }^{[1]}$. Ces auteurs en ont cependant retracé plusieurs, tant publics que privés, qu'ils présentent dans leur livre. Parmi ces jardins, les lieux ouverts au public sont sans doute les mieux placés pour contribuer à la diffusion de cette tradition. Les activités qu'ils proposent devraient refléter cette volonté de faire comprendre aux visiteurs leurs origines et leurs caractéristiques spécifiques. On a donc regroupé une vingtaine de jardins ouverts au public pour former une corporation appelée Association des jardins du Québec (AJQ), dont la mission est « d'aider à leur développement et à leur promotion et de souligner leur apport à la culture et au patrimoine québécois» ${ }^{[2]}$. Malgré l'engouement constaté pour l'horticulture, la fréquentation des jardins publics ne semble pas avoir suivi la tendance ${ }^{[3]}$. Ainsi, les membres de l'AJQ aimeraient unir leurs efforts pour attirer davantage de visiteurs en offrant des circuits thématiques ou des forfaits horticoles à la grandeur du territoire québécois.

Le travail de recherche réalisé a permis de définir des critères de classement des jardins de l'AJQ et de déterminer ceux qui appartiennent au groupe des jardins historiques, tel que présenté au tableau 1. Pour ce faire, une recension des écrits portant sur l'histoire mondiale des jardins a été réalisée, grâce entre autres au livre de Baridon ${ }^{[4]}$, afin d'identifier les types de jardins patrimoniaux représentés. Les catégories de jardins définies à l'échelle mondiale ont fait l'objet d'une adaptation pour refléter le contexte québécois, d'une échelle plus modeste, à l'aide du livre de Martin et Morisset. Une fois les jardins sélectionnés, une revue des pratiques a été réalisée afin d'identifier quels types d'activités éducatives et culturelles sont proposés aux visiteurs. Le but de cette recherche était de répondre à la question initiale, à savoir : le caractère historique d'un jardin influence-t-il la nature de ses programmes éducatifs ? Cette revue a été effectuée par la cueillette d'information sur les sites Internet des institutions, par des visites et des entretiens avec les 
responsables de l'éducation ou des programmes culturels. Les activités offertes ont été répertoriées et classées selon leur nature et leur public cible, afin d'identifier les points forts et les lacunes des programmes.

TABLEAU 1 :

Liste des membres de l'Association des jardins du Québec (ordre alphabétique) en 2005 (Voir page suivante)

Concepts théoriques et définitions

Dans un premier temps, une définition du concept de jardin historique, parfois appelé patrimonial ou ancien, s'impose. Le Comité international pour les jardins et les sites historiques de l'ICOMOS-IFLA ${ }^{[s]}$ a adopté la définition suivante lors de son premier Colloque sur la conservation et la restauration des jardins historiques en $1971:$ « un jardin historique est une composition architecturale et végétale qui présente du point de vue de l'histoire et de l'art un intérêt public " ${ }^{[6]}$. Les travaux de ce comité ont mené à lélaboration, en 1981, de la Charte de Florence relative à la sauvegarde des jardins historiques.

Pour leur part, Martin et Morisset ${ }^{[n]}$ ont établi une liste de critères leur permettant d'identifier les jardins historiques du Québec : ancienneté du tracé d'un jardin (une cinquantaine d'années minimum);

- lisibilité de sa structure, de sa fonction ou de son genre à l'origine;

- présence de plantations, d'aménagements ou de mobilier ancien;

- importance historique du concepteur ou du propriétaire d'origine;

- état présent de conservation.

Le classement des sites patrimoniaux effectué par les gouvernements provincial et fédéral est également une bonne source d'information sur les jardins historiques. Au Québec, la Commission des biens culturels du Québec est chargée d'appliquer la Loi sur les biens culturels (L.R.Q., chapitre B-4) qui mentionne que les sites, plutôt que les bâtiments, devraient recevoir le classement. Le gouvernement du Canada a aussi son système de classement des lieux historiques, sous la gouverne de la Commission des lieux et des monuments historiques du Canada, organisme responsable de désigner les "lieux historiques nationaux du Canada ». En 1994, la commission a exprimé un avis concernant la caractérisation des parcs et des jardins d'importance nationale et inclut maintenant un plus grand nombre de sites naturels.

Une fois que la présence de vestiges anciens de jardins a été confirmée et reconnue, il convient d'adopter des politiques de conservation qui respectent
[5]

L'ICOMOS est le Conseil international des monuments et des sites et l'IFLA est la Fédération internationale des architectes paysagistes.

[6]

Colloque international sur la conservation et la restauration des jardins historiques, Fontainebleau, France, 13-18 sept. 1971, p. 230 (disponible sur Internet : <http://www. international.icomos.org/publications/font28.pdf>)

[7]

MARTIN ET MORISSET, op. cit, p. 30. 


\begin{tabular}{|c|c|c|}
\hline NOM DE L'INSTITUTION & ANNÉE DE CRÉATION DU JARDIN & TYPE DE JARDIN \\
\hline Centre de la nature de Laval & 1971 & Parc floral à vocation récréative / Site historique \\
\hline Domaine de Maizeret & 1849 & Jardin historique \\
\hline Domaine Joly-De Lotbinière & 1851 & Jardin historique \\
\hline Domaine Mackenzie-King dans le parc de la Gatineau & 1903 & Jardin historique \\
\hline Grands Jardins de Normandi & 1996 & Parc floral à vocation récréative. \\
\hline Jardin botanique de Montréal & 1931 & Jardin historique \\
\hline Jardin Daniel A. Séguin & 1995 & Parc floral à vocation pédagogique \\
\hline Jardin du Gouverneur du Musée du Château Ramezay & $\begin{array}{l}\text { Construction du château en } 1705 \\
\text { Reconstitution du jardin en } 2000\end{array}$ & Représentation de jardin historique \\
\hline Jardin botanique Roger-Van den Hende & 1966 & Jardin didactique \\
\hline Jardin Scullion & 1987 & Parc floral à vocation récréative \\
\hline Jardin zoologique du Québec (site fermé en 2006) & 1931 & Parc floral à vocation récréative / Site historique \\
\hline Jardins de Métis & 1926 & Jardin historique \\
\hline Parc Marie-Victorin & 1985 & Jardin commémoratif \\
\hline Maison Henry-Stuart & 1849 & Jardin historique \\
\hline Maison Saint-Gabriel & $\begin{array}{l}\text { Construction de la maison en } 1668 \\
\text { Reconstitution du jardin en } 2000\end{array}$ & Représentation de jardin historique \\
\hline Parc Aquarium du Québec & 1956 & Parc floral à vocation récréative \\
\hline Parc des Champs-de-Bataille / y compris le jardin Jeanne d'Arc & $1908 / 1938$ & Jardin historique \\
\hline Parc du Bois-de-Coulonge & 1840 & Jardin historique \\
\hline Parc Jean-Drapeau & 1967 & Parc floral à vocation récréative \\
\hline Roseraie du Témiscouata & 1997 & Parc floral à vocation récréative/Site historique \\
\hline Seigneurie des Aulnaies & 1853 & Jardin historique \\
\hline Villa Bagatelle & Vers 1850 & Jardin historique \\
\hline
\end{tabular}


l'intégrité du site, tel que défini dans la Charte de Florence. Par contre, lorsque les vestiges d'un jardin ont complètement disparu, il est aussi possible de recréer ce lieu selon deux approches :

1] La reproduction d'un jardin s'effectue sur le site originel, en respectant la superficie initiale et selon les plans disponibles, mais il $\underline{\text { ne reste plus de vestiges matériels du jardin. }}$

2] La représentation est une création selon l'esprit initial d'un jardin qui ne s'effectue pas nécessairement sur l'emplacement original et qui laisse place à l'interprétation. Deux exemples de cette approche, le jardin du Gouverneur du Château Ramezay et le jardin de la Métairie de la Maison Saint-Gabriel, seront considérés à la section suivante.

Le Comité ICOMOS-IFLA a également pris position, lors de son premier colloque, sur les fonctions éducative et culturelle du jardin historique dans les termes suivants : «Le jardin ne doit pas être considéré comme un luxe anachronique. [...] Il faut susciter la connaissance et la compréhension des jardins historiques et éviter de transformer les jardins historiques en parcs de loisirs, les deux fonctions étant inconciliables ${ }^{\left[{ }^{[8]}\right.}$. " Il apparaît donc important de développer des programmes éducatifs de qualité pour faire connaître l'histoire et favoriser une bonne appropriation des sites par les visiteurs.
[8]

Colloque international sur la conservation et la restauration des jardins historiques, op. cit, p. 231-232.

\section{Définition des styles de jardins}

L'histoire des jardins remonte à l'Antiquité où déjà, en Égypte et en Mésopotamie, des vestiges archéologiques de ce que furent les jardins des grands souverains ont été retracés. C'est dire qu'il s'agit là d'une vaste histoire qu'il serait trop long de résumer en entier ici. L'histoire des jardins québécois commence aux premiers temps de la colonie française en Amérique, même si la plupart des jardins représentés au sein de l'AJQ ont été créés aux XIX et $\mathrm{XX}^{e}$ siècles. Afin de définir les styles qui couvrent cette période, la division proposée par Baridon a été utilisée :

- jardins de l'âge baroque (1600 - 1700),

- jardins de l'Homme sensible (1700 - 1790),

- jardins de l'ère industrielle (1800 - 1900),

- jardins de notre temps (1900 - 2000). 
[9]

Pour une description plus détaillée des jardins et de leurs programmes éducatifs, voir PROULX, Ève-Marie. Étude des programmes éducatifs offerts au public dans les jardins historiques du Québec. Rapport de travail dirigé de maitrise (muséologie), Université de Montréal, 2006, p. 24-47.

[10]

MARTIN et MORISSET, op. cit, p. 18.
Martin et Morisset soulignent que l'histoire des jardins anciens du Québec n'est pas encore bien documentée et que leur ouvrage rassemble surtout des témoignages significatifs de ce passé. Ils jettent tout de même quelques balises, évoquées ci-dessous, pour relier l'histoire des jardins québécois aux grands courants mondiaux. Les jardins historiques de l'AJQ retenus pour cette étude s'insèrent dans cette évolution ${ }^{[9]}$.

Les premiers contacts des colons avec la nature remontent à l'arrivée des Français sur le nouveau continent. Selon Martin et Morisset, les trois objectifs des jardins de l'époque de la Nouvelle-France sont la commodité, la science et la beautét ${ }^{[0]}$. De cette période, il reste surtout des traces écrites ou figurées, les vestiges les plus intéressants cités par les auteurs étant les jardins de l'Hôpital-Général de Québec et le jardin des Ursulines de Québec. Par contre, la Maison Saint-Gabriel et le Château Ramezay sont deux sites historiques montréalais qui ont entrepris au début des années 2000 de recréer un jardin de l'époque de la Nouvelle-France pour enrichir leurs activités d'interprétation. Ces deux jardins représentent bien l'âge baroque puisqu'ils contiennent un potager, des plantes aromatiques et médicinales et des plantes d'ornement, présentées dans un agencement structuré.

La deuxième phase de développement des jardins du Québec coïncide avec le changement de régime politique : l'arrivée des Anglais à la fin du XVIII siècle favorise l'essor des "jardins de l'Homme sensible». Comme en Europe, à partir de 1775, les jardins ne sont plus aussi géométriques et systématiques, délaissant la rigueur formelle pour faire place à davantage de liberté. Ils deviennent un espace ouvert sur le paysage environnant, utilisant les forces et les éléments de la nature. Au début du XIX ${ }^{\mathrm{e}}$ siècle, l'aménagement paysager du parc du Bois-de-Coulonge (et quelques années plus tard de la Villa Bagatelle) est imaginé par Henry Atkinson et son jardinier, Peter Lowe, dans le style pittoresque caractérisé par de longues allées recourbées et des massifs floraux qui s'intègrent au paysage.

Au XIX ${ }^{\mathrm{e}}$ siècle, plusieurs mouvements, dont l'industrialisation, favorisent l'expansion des formes de jardins. La villégiature suburbaine et estivale est rendue possible, à la suite du développement des moyens de transport fluviaux et ferroviaires. Les seigneurs, propriétaires terriens progressistes, créent de grands domaines paysagers dont deux exemples sont présents au sein de l'AJQ, soit le Domaine Joly-De Lotbinière et la Seigneurie des Aulnaies. La partie historique du domaine de Maizerets, aménagée au milieu du XIX ${ }^{e}$ siècle par les prêtres du Séminaire de Québec, est aussi représentative de cette époque.

La période du début du XX $\mathrm{XX}^{\mathrm{e}}$ siècle est la plus représentée dans les jardins anciens du Québec. C'est une époque où l'on assiste à une grande liberté, où les règles sont moins strictes. Les jardins font la synthèse des réalisations 
antérieures : «Chacun peut alors exprimer sans contrainte son rapport à la nature, réinterpréter le jardin italien, évoquer le jardin régulier, le jardin clos ou le jardin paysager ${ }^{[11]}$. " À partir de 1920, les Jardins de Métis ont été créés et entretenus par $\mathrm{M}^{\mathrm{me}}$ Elsie Reford. Ils sont maintenant renommés pour leur créativité, des collections uniques et l'intégration de plantes rares grâce à un microclimat favorable. Les jardins de la Maison Henry-Stuart à Québec ont également été élaborés à cette époque.

À la fin du XIX ${ }^{\mathrm{e}}$ siècle et au début du XX' $\mathrm{XX}^{\mathrm{e}}$ siècle, les mêmes phénomènes qu'en Europe sont observés, soit la présence de jardins d'ouvriers, le développement des banlieues et le phénomène des cités-jardins. Ces mouvements entraînent la création du parc des Champs-de-Bataille en 1908. Aménagé par le célèbre architecte paysagiste Frédérick Todd, il s'est par la suite enrichi du jardin Jeanne d'Arc en 1938. Au début du XX' siècle, des représentants de la classe politique s'intéressent à l'horticulture, dont le premier ministre du Canada, William Lyon Mackenzie King. Son domaine intègre plusieurs courants horticoles de différentes époques, comme des plates-bandes symétriques, des ruines gothiques et des massifs fleuris plus modernes.

Dans les années 1930, alors que la crise économique fait rage, le frère Marie-Victorin supervise la création du Jardin botanique de Montréal. Ce jardin à vocation éducative, qui a fêté son $75^{\mathrm{e}}$ anniversaire en 2006, possède maintenant une importante collection de plantes, réparties dans dix serres d'exposition et une trentaine de jardins thématiques. Au Québec, comme ailleurs dans le monde, la Deuxième Guerre mondiale crée une coupure dans l'évolution des jardins. Dans les années 1950, on assiste à une démocratisation de l'horticulture et à un éclatement des styles qui a mené à la multiplication des jardins privés et à de grands événements populaires comme les Floralies de Montréal en 1980 et les expositions récentes des Mosaïcultures au Vieux-Port.

Analyse des programmes éducatifs des jardins historiques

\section{Caractéristiques du public et des programmes éducatifs des jardins}

Comme champ d'études, les jardins sont peu documentés. Selon Valérie Gaudreau $^{[12]}$, il n'y a jamais eu au Québec de sondage national permettant de définir les caractéristiques du public des jardins. On sait toutefois que ce public est constitué majoritairement de la génération des "baby-boomers", férue d'horticulture. Même dans un pays comme la Grande-Bretagne,
[11]

MARTIN et MORISSET, op. cit, p. 28.

[12] GAUDREAU, Valérie, "Grands jardins, vives passions *. Continuité, no 105 , été 2005, p. 22. 
[13]

CONNELL, J.

"Managing Gardens for Visitors in Great Britain: A Story of Continuity and Change Tourism Management, no 26, 2005, p. 185-201; et « The Purest of Human

Pleasures: The Characteristics and Motivations of Garden Visitors in Great Britain ». Tourism Management, $n^{\circ} 25$, 2004, p. 229-247.

[14]

O'NEIL, M.-C. « Discours de l'exposition, discours du programme éducatif . In. ALLARD, M. et B. LEFEBVRE. Musée, culture et éducation. Québec : Éditions MultiMondes, 2000, p. 33-42 qui possède un grand nombre de jardins historiques ouverts au public, les données concernant les visiteurs sont peu nombreuses. Une étude récente, réalisée par Connell[ ${ }^{131}$, tente de pallier cette lacune. Selon cette étude, $60 \%$ des visiteurs de jardins ont un intérêt général pour le jardinage, mais seulement $10 \%$ d'entre eux possèdent des connaissances spécifiques en horticulture.

Les programmes éducatifs développés dans les jardins devraient donc s'adresser principalement à un public d'adultes ayant un intérêt général pour l'horticulture. La nature des programmes éducatifs muséaux destinés aux adultes est relativement peu documentée puisque les recherches dans ce domaine privilégient souvent le public scolaire. On distingue plusieurs types d'activités liées au musée, entourant la visite des expositions permanentes et temporaires. Ces activités peuvent être classées en fonction des rapports spatiaux et intellectuels qu'elles entretiennent avec l'exposition et en fonction des liens entre les différents programmes proposés. Le moment où est effectuée l'activité par rapport à la visite du site peut aussi être étudié pour élaborer des activités préparatoires et de prolongation. Ces critères, définis par les recherches d'O'Neil ${ }^{[14]}$, ont servi de base pour la comparaison des programmes. À la section suivante, les activités ayant un lien intellectuel direct avec la thématique centrale des jardins seront abordées, puis les activités de type culturel, qui se distinguent ou non de la mission première d'interprétation des institutions, seront discutées.

\section{Type d'information à transmettre aux visiteurs}

Un aspect primordial de l'interprétation dans un jardin est le contact primaire du visiteur avec le site, la perception émotive découlant de ce qu'il voit et de ce qui l'inspire une fois qu'il a terminé sa visite. Pour comprendre "l'esprit du lieu», le visiteur doit pouvoir " lire un paysage», une activité intellectuelle qui n'est pas intuitive. Les outils d'interprétation mis à sa disposition doivent lui permettre d'effectuer cette lecture en proposant des clés pour mieux comprendre et apprécier le type d'aménagement du jardin, sa structure et son style. Des outils doivent aussi servir à identifier le type de végétaux présents. En plus des renseignements horticoles, l'interprétation peut porter sur l'histoire du site et de ses fondateurs, de même que sur les étapes de restauration et les problèmes de conservation du jardin. Le défi qui est posé pour que l'expérience de visite soit réussie est de faire le lien entre l'horticulture et l'histoire du site. Les différentes options d'interprétation à la disposition du visiteur sont maintenant considérées. 


\section{Moyens d'interprétation personnalisés ou non?}

Les moyens d'interprétation offerts doivent respecter l'ambiance champêtre et agréable des jardins ; c'est pourquoi la nécessité d'offrir des visites guidées ne fait pas l'unanimité auprès des responsables consultés. Certains pensent qu'elles ne sont pas essentielles en raison de l'attitude contemplative développée lors de la visite d'un jardin, alors que d'autres considèrent que c'est la seule façon de faire comprendre aux visiteurs les attributs particuliers de leurs plantations. Le visiteur préférant faire une visite autonome dispose de différents moyens, tels que le plan du site, le livret de visite, les plaquettes d'identification des végétaux, les expositions et les panneaux d'interprétation.

Pour faire une visite autonome, le plan du site semble être un élément essentiel, remis aux visiteurs dès leur arrivée ou, pour les plus petits sites, disponible sur un panneau d'interprétation dans le jardin. Selon Walker ${ }^{[15]}$, seulement $2 \%$ des visiteurs prendraient le temps de lire sur place l'information contenue dans un guide de visite, qui constitue davantage un souvenir qu'un outil efficace de visite.

Certaines institutions innovent en ayant recours à des technologies audiovisuelles comme les bornes d'information sur les plantes offertes au jardin Jeanne d'Arc et au jardin du Château Ramezay. Ce moyen novateur demeure assez limité puisque, dans le cas du jardin Jeanne d'Arc, il fournit de l'information à caractère horticole seulement. Un autre outil technologique intéressant, mais encore peu exploité par les jardins, est l'audio-guide. L'amélioration des technologies diminuera sans doute les coûts de production et d'entretien de ce genre de service d'ici quelques années. Toutefois, il peut aussi nuire à l'aspect contemplatif de la promenade, en forçant le visiteur à écouter une grande quantité d'information. Même s'il ne correspond pas aux besoins de tous les visiteurs, comme le mentionnent Lefebvre et Lefebvre dans une recherche sur l'utilisation d'un audio-guide dans les serres du Jardin botanique de Montréal[16], il constitue probablement une option intéressante à développer.

L’identification des végétaux peut donc se faire de différentes manières, plus ou moins discrètes selon l'effet désiré. Toutefois, les renseignements fournis ne doivent pas se limiter aux noms des plantes, qui ne contribuent pas beaucoup à enrichir les connaissances d'un visiteur non averti. Le contenu des outils d'interprétation non personnalisés doit être constitué d'un mélange de renseignements horticoles, historiques et architecturaux.

Les institutions qui disposent de salles d'expositions adjacentes aux jardins offrent des expositions qui font le lien entre l'intérieur et l'extérieur. Celles-ci permettent souvent de raconter l'histoire des habitants
[15]

WALKER, V. « Interpreting the Past for the Present and the Future *. Téoros. Printemps 1999 , p. 43.

[16] LEFEBVRE, B. et H. LEFEBVRE. «Le visiteur, le guide et l'éducation *. Revue canadienne de l'éducation, vol. 16, no 3, 1991 , p. 335. 
du site, puisque ce sujet n'a pas forcément besoin d'être abordé à l'extérieur. Des expositions temporaires peuvent élargir le contenu intellectuel du jardin en éveillant le visiteur à différents aspects de l'histoire de l'horticulture. L'exposition Aventures botaniques, présentée au Château Ramezay à l'été 2005, était un bon exemple du type de liens qui peuvent être créés entre le jardin et le musée puisqu'elle relatait les façons de transporter, de conserver et de cultiver les plantes du Nouveau Monde en France.

En général, les institutions offrent des visites guidées de contenu standard au public et aux groupes. Le contenu des visites peut varier en fonction du type de visiteurs, puisque certaines institutions répondent aux demandes des groupes ayant des intérêts spécifiques, comme les membres d'une société d'horticulture, en mettant un spécialiste à leur disposition. Par contre, pour les visites offertes au grand public, les institutions ont recours à des guides qui transmettent une quantité d'information uniforme.

La formation des guides peut aussi influencer leur capacité à fournir de bons renseignements au public. Selon les réponses obtenues dans cette étude, le profil universitaire des guides qui est recherché est davantage lié aux sciences humaines qu'aux sciences biologiques. Cela peut poser un problème pour certaines institutions qui engagent des étudiants avant tout pour leurs connaissances en histoire ou en politique et qui éprouvent des difficultés à intéresser ces jeunes à l'horticulture. Pour pallier ce manque, certaines demandent aux jardiniers de répondre aux questions des visiteurs liées à l'horticulture. D’ailleurs, en Angleterre, pays où la visite de jardins historiques est plus développée, une étude récente démontre que les jardins offrant des moyens d'interprétation à la fine pointe de la technologie audiovisuelle sont peu nombreux ${ }^{[17]}$. L'étude mentionne qu'il s'agit peut-être d'un choix pour maintenir le caractère naturel des sites et privilégier les contacts humains. Elle révèle aussi qu'un des aspects préférés des visiteurs est la possibilité d'être mis en contact avec les jardiniers qui travaillent sur le site. Cette approche humaine a été adoptée par certains jardins québécois, comme celui du domaine Mackenzie-King, qui utilise les services de dames retraitées et bénévoles pour effectuer cette tâche, et devrait être exploitée davantage par les autres jardins puisqu'elle correspond à un besoin exprimé par les visiteurs. Ainsi, le modèle classique des visites guidées répond bien à la demande lorsqu'il s'agit de transmettre l'information historique du site et la présence de personnes-ressources postées dans les jardins est un moyen de favoriser la diffusion de l'information horticole.

Par ailleurs, la question des programmes éducatifs pour les jeunes dans les jardins historiques a été abordée avec les responsables, puisqu'il semble que ce thème ne se prête pas facilement aux visites scolaires. D'abord, la saison estivale n'est pas favorable pour ces visites puisque les étudiants sont en vacances. De plus, il est risqué de planifier des visites en raison des 
aléas de la température. Par ailleurs, il semble que les enfants ne soient pas très sensibles au caractère esthétique et contemplatif des jardins. Les institutions qui attirent les groupes scolaires offrent des visites portant sur la conservation de la faune et de la flore, un thème qui correspond davantage aux goûts des enfants et aux exigences du programme de formation. Par contre, on peut initier les jeunes au jardinage, une activité concrète et stimulante, comme c'est le cas au Jardin botanique de Montréal qui, depuis sa fondation, est l'institution modèle pour ce genre d'activité.

\section{Activités spéciales et culturelles}

Ces activités sont regroupées parce qu'elles ont un lien intellectuel indirect avec les thématiques du site, même si elles sont parfois choisies en fonction de la mission de celui-ci. La plupart des jardins historiques présentent des concerts, des pièces de théâtre et d'autres activités qui permettent de renouveler la clientèle en attirant de nouveaux visiteurs. Ainsi, dans le cas des séries de conférences dominicales de la Maison Saint-Gabriel, il s'agit de programmer un retour des visiteurs intéressés par l'horticulture. Même si l'étude de Connell a révélé que les événements ne sont pas d'importance primordiale pour les visiteurs ${ }^{[18]}$, ils restent une bonne façon d'attirer l'attention des médias. La plupart des institutions mentionnent choisir leurs activités en fonction de leur caractère culturel, un créneau touristique en développement. L'exemple des Jardins de Métis, qui a créé un Festival international de jardins, est remarquable. Cet événement culturel amalgamé au jardin historique, qui se déroule chaque année depuis 2000 , a acquis une réputation internationale et attire les spécialistes et les amateurs d'art contemporain. Il semble donc que ce mariage entre nature, culture et histoire favorise la mise en valeur des sites patrimoniaux et corresponde aux besoins du public.

\section{Évolution des programmes éducatifs dans les jardins}

Une comparaison des types d'organismes gérant les sites permet de constater que seulement le quart des institutions historiques sont ouvertes au public depuis plus de vingt-cinq ans, la moitié ont ouvert leurs portes dans les années 1980 et le quart ne sont ouvertes que depuis quelques années. Il est évident que les plus récents programmes éducatifs sont toujours en évolution. De plus, les modes de gestion et les moyens financiers des institutions sont très variables. L'affiliation à des organismes gouvernementaux, comme dans le cas du parc du Bois-de-Coulonge qui peut désormais compter sur les services 
des spécialistes de la Commission de la Capitale nationale du Québec, est favorable à l'amélioration de la qualité des activités proposées. Toutefois, l'aspect horticole n'est pas le seul à primer au sein d'organismes voués à la commémoration historique. Ainsi, le jardin peut parfois se trouver noyé dans une grande quantité d'activités non reliées, comme c'est le cas à la Commission des champs de bataille, qui ne met pas au premier plan l'aspect botanique dans l'élaboration de ses activités éducatives. Par ailleurs, lorsque le site a une vocation récréative, les aspects historiques peuvent être occultés et l'utilisation extensive du site peut même contribuer à sa dégradation. Il importe donc de maintenir un bon équilibre entre la vocation du site, la commémoration et la conservation de ses artéfacts historiques. Finalement, la reconstitution de jardins par des sites historiques semble être la tendance la plus récente. Les musées qui ont créé ces jardins affirment qu'ils constituent des outils pédagogiques innovateurs. Ils s'en servent pour développer des activités éducatives nouvelles qui les positionnent dans un créneau différent des autres institutions historiques.

\section{Impact de l'Association des jardins du Québec}

Il existe peu de travaux publiés pour élaborer une histoire des jardins du Québec. Certains ouvrages récents, comme le livre de Reford sur les jardins oubliés de $1860-1960^{[19]}$, contiennent d'admirables photographies de jardins anciens, mais ne documentent pas de façon formelle leur histoire. Les recherches spécifiques à chacun des jardins doivent être poursuivies pour mieux les situer les uns par rapport aux autres. De plus, certains jardins de moins de cinquante ans méritent sans doute d'être inclus dans la catégorie des jardins historiques, puisque leur statut est parfois menacé par le développement immobilier ou récréotouristique. Ainsi, le parc Jean-Drapeau et le jardin Roger-Van den Hende pourraient être considérés comme faisant partie du patrimoine horticole et ils pourraient être mis en valeur en fonction de cette reconnaissance. Il est également dommage que d'autres jardins historiques importants, comme ceux du domaine Cataraqui à Sillery et du domaine Howard à Sherbrooke, ne soient plus membres de l'AJQ. Pour obtenir un véritable impact, l'AJQ devrait représenter un plus grand nombre de sites afin d'offrir un portrait complet des jardins québécois. Du côté des jardins privés, il existe au Québec des sites très intéressants, en particulier les jardins de Quatre-Vents, dans Charlevoix. Les jardins du Séminaire de Saint-Sulpice de Montréal mériteraient également davantage d'attention, car ils comptent parmi les plus vieux jardins au Canada à avoir subsisté jusqu'à nos jours. La possibilité de créer des liens entre les jardins privés et les jardins publics à travers l'AJQ devrait être envisagée. En plus de favoriser la 
visibilité des sites par la publication d'un guide annuel, l'AJQ est un excellent outil de promotion des activités ponctuelles, diffusées sur son site Internet. Par contre, avec ses moyens financiers limités, cette association n'est pas suffisamment présente dans les médias, même si le jardinage compte de nombreux adeptes au Québec.

\section{Conclusions}

À la suite à cette analyse, il est clair que le caractère historique des jardins influence les programmes proposés. Les institutions membres de l'AJQ offrent toutes des moyens d'interprétation comme des panneaux d'interprétation et un plan du site. De nouveaux outils, notamment des bornes interactives et des audio-guides, mériteraient d'être développés afin d'offrir davantage d'information aux visiteurs tout en respectant leur autonomie. Quant aux visites guidées, leur contenu doit allier histoire et horticulture afin de transmettre aux visiteurs des clés qui favorisent la compréhension de l'organisation spatiale du site. Différentes activités, de nature artistique ou sociale, viennent compléter l'offre touristique et confirment la vocation culturelle des sites. Le développement des programmes éducatifs devrait s'accentuer dans les prochaines années, puisque la reconnaissance des jardins historiques est très récente au Québec. L'interprétation dans les jardins commence à intéresser les muséologues et les responsables des sites. De récentes collaborations entre l'Université de Montréal, l'École du Louvre et le Château Ramezay contribuent à élaborer la recherche dans ce domaine novateur, alliant nature, culture et histoire. En favorisant la réalisation de projets de diffusion et d'éducation de qualité, les jardins historiques pourront être reconnus et appréciés pour les grandes richesses qu'ils contiennent. 


\section{Summary}

\section{A Study of Educational Programs Offered to the Public}

in the Historic Gardens of Quebec

[translated by Allana Carlyle]

This research examined the educational programs offered by the historic public gardens of Quebec belonging to the Quebec Gardens' Association (QGA). The selection of these gardens was based on the desire of the QGA representatives to educate the public on the little-known reality of historic gardens. To accomplish this, the association chose to develop the historical and educational aspects of these

[20]

BARIDON, Michel.

Les jardins : paysagistes, jardiniers, poètes, Laffont, Paris, 1998, 1239 pages.

[21]

MARTIN, Paul-Louis et Pierre MORISSET, Promenades dans les Jardins Anciens de Québec,

Photographies de Janouk

Murdock, Les Éditions du

Boréal, B.L. Éditeur, Montréal, 1996, 177 pages. gardens in order to help the public acquire the background knowledge necessary to enrich their visits of the participating institutions.

The first stage of the research was to define the criteria necessary for the categorical classification of the QGA gardens with the goal of identifying those gardens considered to be historic. To achieve this, a literature review on the world history of gardens was completed, largely based on the work of Michel Baridon ${ }^{[20]}$. The goal of this review was to identify, more specifically, the types of heritage gardens represented within the association. The categories of gardens defined on a world scale were then adapted, with the help of Paul-Louis Martin and Pierre Morisset's work on the ancient gardens of Quebec, to reflect the more modest regional context of Quebec ${ }^{[21]}$.

Once the gardens were selected and classified, a review of the gardens' practices was completed in order to identify the activities being offered to visitors of these institutions. The goal of this step was to answer the original question of whether or not the historical character of a garden influences the nature of the educational programs offered its visitors.

The review was completed through the collection of information from the institutions' websites and through personal visits. Afterwards, the directors of education and cultural programming for these gardens were interviewed to gain a deeper understanding of their methods of information distribution. All types of activities were included in this research, including programs designed for school groups. The results were then recorded and classified in order to propose new interpretive and educational strategies for the QGA to integrate into their programming. 
The results of this study revealed many positive elements regarding the transmission of historical information to the visitors. For example, guided tours often open a window to the history of a particular site and its occupants. Maps of the sites generally contain a short historical description and interpretive signs combining horticultural, historical and architectural information help to present the garden to its visitors. In short, the historic character of the gardens is utilised and emphasised through a series of interpretive means which encourage the understanding of the cultural significance of historic gardens. Other activities of an artistic or social nature complete the tourist experience and combined, confirm the cultural importance of these institutions as well as their dedication to cultural tourism. However, one of the concerns brought up during this study is the lack of connection between historical and horticultural data, particularly during guided tours, often lead by guides with backgrounds in history rather than botany. The comparison of the variety of programs offered by these institutions allowed, among other things, the conclusion that the gardens' interpretive programs are in early stages of development and will continue to be enriched throughout the coming years.

In the light of the results of this study, it is suggested that research work in museology continue so as to support the development and diffusion of historic gardens through innovative interpretation. 